\title{
Design, manufacture and characterization of an optical fiber glucose affinity sensor based on an homogeneous fluorescence energy transfer assay system
}

\author{
D.L. Meadows ${ }^{1}$ and J.S. Schultz ${ }^{2}$ \\ Department of Chemical Engineering, The University of Michigan, Ann Arbor, MI 48109 (USA)
}

(Received 5th November 1992; revised manuscript received 16th January 1993)

\begin{abstract}
Optical fiber biosensors based on fluorescence assays have several distinct advantages when measuring biological analytes such as metabolites, cofactors, toxins, etc. Not only are optical signals immune to electronic interferences, but the polychromatic nature of most fluorochemical assays provides more potentially useful data about the system being studied. One of the most common difficulties normally encountered with optical biosensors is the inability to routinely recalibrate the optical and electronic components of the system throughout the life of the sensor. With this in mind, an optical fiber biosensor system for glucose has been constructed along with the peripheral electronic instrumentation. The biochemical assay is based on an homogeneous singlet/singlet energy transfer affinity assay. The sensor probe indirectly measures glucose concentrations from the level of fluorescence quenching caused by the homogeneous competition assay between TRITC labeled concanavalin A (receptor) and FITC labeled Dextran (ligand). The FITC signal is used as an indicator for glucose concentrations and the TRITC signal is used for internal calibration. Chemical derivatization procedures using succinic anhydride were developed to prevent aggregation of the receptor protein in solution, and the TRITC/ConA ratios were optimized to achieve the best sensor performance. Using this sensor system, the FITC-Dextran detection limit was $0.05 \mu \mathrm{g} / \mathrm{ml}$ and glucose concentrations up to $1600 \mathrm{mg} / \mathrm{dl}$ could be detected with a time response of approximately $10 \mathrm{~min}$.
\end{abstract}

Keywords: Biosensors; Concanavalin A; Fluorescence energy transfer; Glucose; Optical fibre biosensors

Biosensors are analytical devices that respond reversibly and specifically to the concentration or activity of a biologically important analyte. Several good reviews on biosensors have recently been published [1-6] that summarize the key developments in biosensor research. During the past several years sensor systems have been re-

Correspondence to: D.L. Meadows, Department of Pharmaceutical Research, Allergan Inc., 2525 Dupont Dr., Irvine, CA 92715 (USA).

1 Present address: Department of Pharmaceutical Research, Allergan, Inc., 2525 Dupont Dr., Irvine, CA 92715 (USA).

2 Present address: Center for Biotechnology and Bioengineering. University of Pittsburgh, 911 William Pitt Union, Pittsburgh, PA 15260 (USA). ported in the literature that are capable of measuring analytes in most of the chemical classifications important to biological systems, e.g. amino acids, cofactors, carboxylic acids, alcohols, inorganic ions, etc., in both aqueous and gaseous phases.

The application of photometric assay techniques such as fluorescence, absorbance, chemiluminescence, internal reflection spectroscopy, etc., to the biosensing area has rapidly grown over the past decade. Good reviews on the subject have been prepared by Saari [7] and Wolfbeis [8]. There are several inherent advantages to an optical fiber based sensor over an electrical based sensor, e.g., isolation from electromagnetic noise 
sources, small size, use in long-distance telemetry, and the availability of spectral data [9].

In an earlier article [10], we reported on the initial results obtained with an assay system for glucose that was based on an homogeneous fluorescence energy transfer assay. The assay was developed for use in an optical fiber biosensor with geometry similar to that developed by Mansouri and Schultz $[11,12]$. They immobilized a glucose receptor, concanavalin $\mathrm{A}(\mathrm{Con} \mathrm{A})$, to the inner wall of a dialysis fiber (diameter $=0.3 \mathrm{~mm}$ ), and used fluorescein labeled dextran (FITC-Dextran) as a macromolecular ligand competing with glucose. The current sensor is based on an homogeneous fluorescence energy transfer assay and is designed to improve upon the Schultz and Mansouri sensor by eliminating the immobilization of ConA, shortening the sensor response time, and correcting for drifts in sensor components by internal calibration.

The current approach employs two labels that absorb and fluoresce visible light, FITC-Dextran as a donor (fluorescer) and tetramethylrhodamine isothiocyanate labeled ConA (TRITCConA) as the acceptor (quencher) [13-15]. The competing reactions for the system are given in Eqns. 1 and 2.

$$
\begin{aligned}
& \text { Glucose + TRITC-ConA } \leftrightarrow \\
& \text { (analyte) (receptor) } \\
& \text { Glucose : TRITC-ConA } \\
& \text { (analyte : receptor) }
\end{aligned}
$$

$$
\begin{aligned}
& \underset{\text { (competing analyte) }}{\text { FITC-Dextran }}+\underset{\text { (receptor) }}{\text { TRITC-ConA } \leftrightarrow} \\
& \text { FITC-Dextran : TRITC-ConA } \\
& \text { (competing analyte : receptor) }
\end{aligned}
$$

When FITC-Dextran reversibly binds to TRITCConA, the donor and acceptor chromophores are sufficiently close together (less than approximately $50 \AA$ ) for a significant portion of the fluorescein signal to be quenched by rhodamine. As glucose is added to the system, FITC-Dextran is liberated from the TRITC-ConA causing the fluorescein signal to increase. With this approach, the fluorescein signal is used to monitor glucose concentrations, and the TRITC signal is used for internal calibration to correct for optical and electronic drift.

The FITC/TRITC chromophore pair was selected because of the high energy transfer efficiency due to the large spectral overlap between the emission spectra of FITC and the absorbance spectra of TRITC. The absorbance and emission spectra are given in Fig. 1 for both FITC and TRITC, and the spectral overlap is indicated by the hatched region. By appropriately selecting the chromophore pair, one can increase or decrease the spectral overlap in order to optimize the efficiency of resonance energy transfer for a particular application [14].

In our most recent work, we have synthesized new TRITC-ConA conjugates that have improved the stability and dynamic range of homogeneous assays when compared with the initial work that had utilized commercially prepared ConA conjugates [10]. Synthesis of these protein conjugates successfully eliminated all ConA aggregation through chemical modification with succinic anhydride followed by labeling with TRITC. The dynamic range of assays using these new TRITCConA conjugates was significantly improved because of the higher dye to protein ratios. An optical and electrical instrumentation system was also constructed for evaluating the sensor perfor-

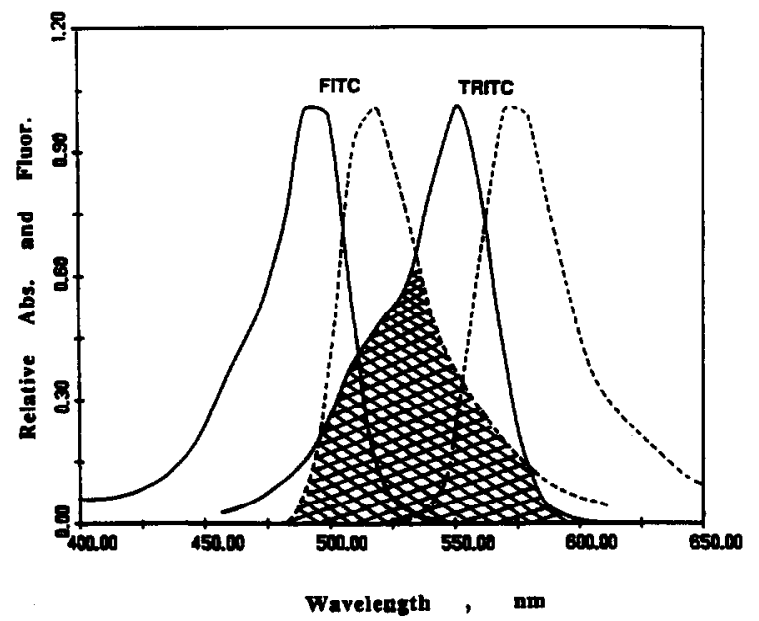

Fig. 1. Spectral overlap of FITC and TRITC. The absorbance spectra are denoted by solid lines and the emission spectra are denoted by dashed lines. The hatched region represents the spectral overlap area. 
mance under various environmental conditions $[16,17]$.

\section{EXPERIMENTAL}

\section{Materials}

Supplies of commercially prepared ConA derivatives from Sigma (St. Louis, MO) were compared against samples of chemically derivatized ConA that were prepared and purified in our laboratory. Commercially prepared samples of TRITC-ConA and succinylated ConA labeled with TRITC (TRITC-Succ-ConA) were supplied in a lyophilized form and were not purified prior to use. FITC labeled dextran, MW $7 \times 10^{4}$, was used as received from Sigma, Pharmacia (Piscataway, NJ), and Molecular Probes (Eugene, OR).

\section{Equipment}

An SLM (Urbana, IL) SPF-500C spectrofluorometer was used for all fluorescence measurements, and a Varian (Palo Alto, CA) DMS 200 UV-visible spectrometer was used to collect all absorbance spectra.

An instrumentation system, shown in Fig. 2, was assembled to test the homogeneous assay system in an optical fiber sensor configuration. The system was capable of multiplexing two excitation wavelengths into optical fibers that guided the light to the assay solution in the sensor probe. Fluorescence signals were collected from the sensor and carried to photon counting PMTs (multialkali photocathode with detection range 185-850 nm; Products for Research, Danvers, MA). Long pass barrier filters $(50 \%$ cut off at $480 \mathrm{~nm}$ and $520 \mathrm{~nm}$ : Oriel Optics, Stratford, CT) were placed just in front of the PMTs to filter out undesired

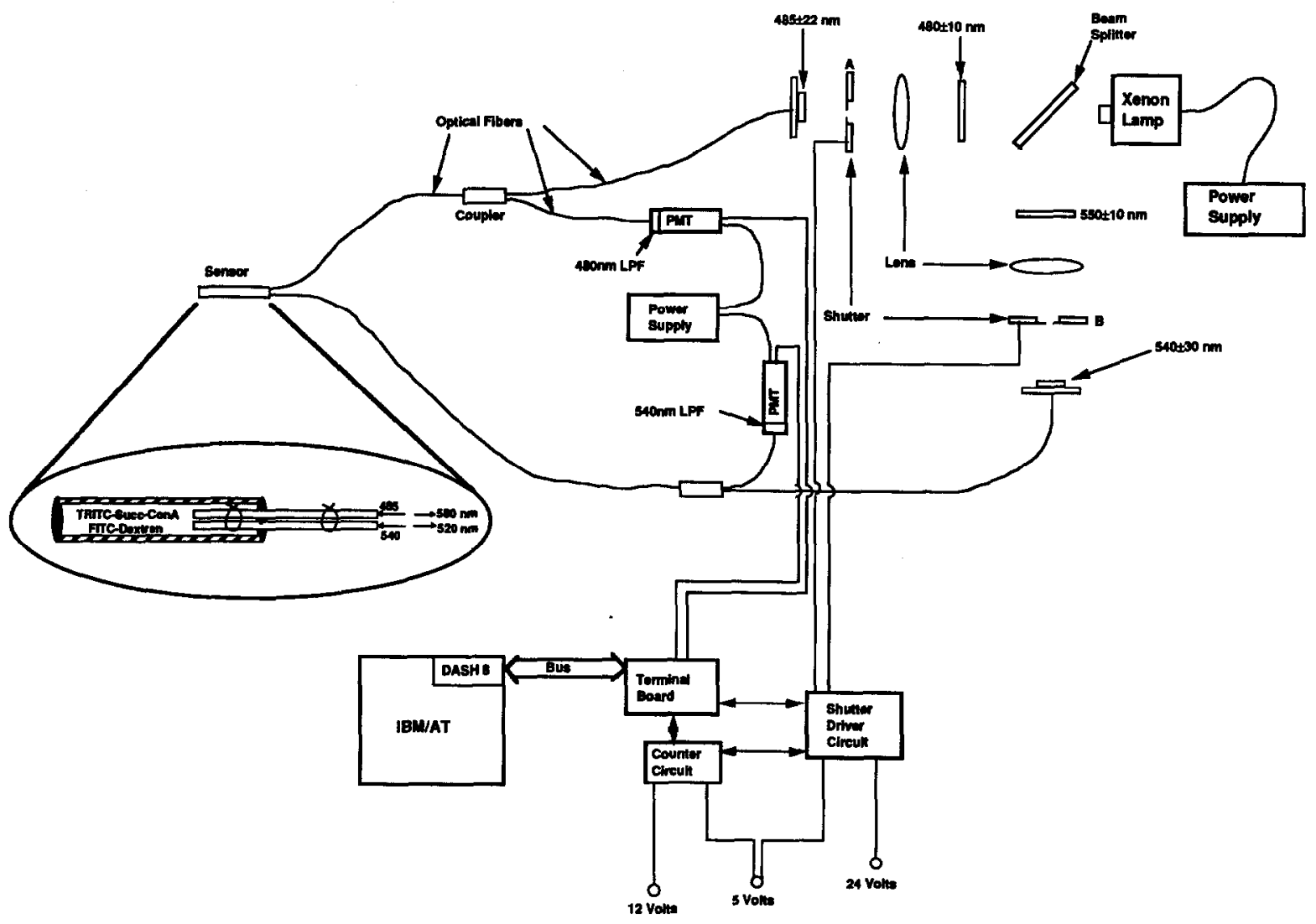

Fig. 2. A schematic diagram of the dual optical fiber sensor system. 
wavelengths of light. White light from a 250 Watt Ealing Electro-Optics (South Natick, MA) xenon light source was passed through a $5 \mathrm{~cm}$ diameter plate beam splitter (50:50 split ratio: Melles Griot, Irvine, CA), then through a $2^{\prime \prime} \times 2^{\prime \prime}$ interference filter (center wavelength $480 \mathrm{~nm}$ and 550 nm with FWHM of $10 \mathrm{~nm}$; Oriel Optics). Two, 5 $\mathrm{cm}$ diameter, $10 \mathrm{~cm}$ focal length, double convex lenses (Melles Griot) focused each beam to the optical fiber mounts. One inch aperture electronic shutters (Melles Griot) were placed between the focusing lenses and the optical fiber mounts. The shutters were controlled by an IBM/AT microcomputer and were connected to a $24 \mathrm{~V}$ and a $5 \mathrm{~V}$ power supply. $24 \mathrm{~V}$ at $3 \mathrm{~A}$ for 8 ms were required to open the shutters, then the shutter driver circuits switched the supply voltage to $5 \mathrm{~V}$ to keep the shutters open. When the voltage was dropped to $0 \mathrm{~V}$, the shutters closed. Just prior to entering the optical fibers, the light was filtered again using 1 in. diameter interference filters (center wavelengths $485 \mathrm{~nm}$ and 540 nm, FWHM $22 \mathrm{~nm}$ and $30 \mathrm{~nm}$ : Omega Optical; Brattleboro, VT). The two beams of light were guided to the sensor using $1: 1$ and $1: 15$ couplers from Canstar (Scarborough, Ont.). All fibers were multimode, graded index, with $100 \mu \mathrm{m}$ core and $140 \mu \mathrm{m}$ cladding. All connections were made using Amphenol 906 connectors (Lisle, IL). Hollow fiber polysulfone membranes were from AG Technologies (Needham, MA) with $0.5 \mathrm{~mm}$ inner diameter and 10000 molecular weight cut off. The optical fiber and hollow fiber were glued together using water activated 910 Adhesive (Permabond International, Englewood, NJ).

In summary, the sequence of events during the data acquisition process was as follows. First, shutter A was opened for a prescribed amount of time. During this interval, the excitation light intensity and the FITC fluorescence signals were measured by the two PMTs. Then shutter A was closed. Next, shutter B then opened for a prescribed amount of time and the excitation intensity and the TRITC fluorescence signal were measured. Then shutter B was closed. The cycle was repeated as many times as the experiment duration permitted. After the experiment was completed, the software computed the average and standard deviation for each sampling interval and stored the data for further manipulation. The excitation intensities were used to normalize the fluorescence signals, while the TRITC signal was used to adjust for drifts in the instrumentation hardware with time. All of the data acquisition procedures were automated using a DASH-8 (Metrabyte, Taunton, MA) A/D card and an IBM/AT microcomputer to direct all of the required steps in the proper sequence. The software was written in Basic and was designed so the operator would input only the period, the duty cycle of the shutters, and the duration of the experiment.

\section{Assembly procedures}

The first step in assembling the sensor probe was to smoothly cleave the ends of the two optical fibers that were to be inserted into the hollow fiber. The ends of the exposed fibers were then lined up flush with each other and carefully tied together with No. 00 suture thread under a dissecting microscope. A 27 gauge needle was connected to a $5 \mathrm{~cm}^{3}$ syringe containing assay solution (TRITC-Succ-ConA/FITC-Dextran). The needle was inserted into the lumen of a one inch long section of hollow fiber. The fiber was filled with assay solution and sealed immediately at one end with cyanoacrylate adhesive. The two optical fibers were quickly inserted into the upper end of the hollow fiber and adhesive applied. The assembled sensor was then submerged in $0.05 \mathrm{M}$ phosphate buffer saline at pH 7.4 (PBS) for at least one hour till the adhesive set.

Response of the sensor to various glucose concentrations was determined by first inserting the sensor into an agitated solution of PBS at $25^{\circ} \mathrm{C}$ to determine a baseline fluorescence signal. Then the sensor was inserted into solutions containing increasing amounts of glucose and the fluorescence signal response was monitored for ten minutes for each solution. After completion of this procedure, the sensor was inserted into a fresh solution of PBS so the sensor signal could return to baseline.

\section{Protein derivatization}

The jack bean lectin protein concanavalin A (ConA) was used as the receptor for the assay 
system. Native ConA is composed of four protomers each having a molecular weight of 26000 Daltons (pConA), and each protomer has one non-interacting sugar binding site. Acetylation or succinylation of the e-amino groups on the lysine residues of ConA can cause the dimer/tetramer equilibrium to shift to the point where tetramers are never formed, even at a $\mathrm{pH}$ greater than 7 and a temperature higher than $25^{\circ} \mathrm{C}$ [18]. The overall reactivity of ConA toward simple sugars and polysaccharides is not significantly affected by these procedures [19-21]. Utilizing this fact, a protein stabilization procedure for preventing ConA aggregation was developed based on derivatization with succinic anhydride followed by labeling with TRITC [16]. Since a dynamic equilibrium normally exists between the dimer and tetramer forms of ConA, it was essential that the dimers of ConA be prevented from aggregating in the lumen of the dialysis fiber. If aggregation was allowed to occur, then precipitation of ConA in the fiber lumen would cause rapid failure of the sensor. By reacting the lysine residues with succinic anhydride, the positive charged lysine side chains were converted to negative charges. These changes in the overall charge distribution of the protein, combined with changes in the steric interactions, caused the native ConA tetramer molecule to separate into two identical dimers that did not re-aggregate into the tetramers even at elevated temperatures. The procedures used for determining the degree of succinylation and TRITC labeling are presented elsewhere [16,22].

\section{Fluorescence quenching}

The excitation and emission wavelengths for measuring fluorescein quenching of the homogeneous energy transfer assay were $480 \mathrm{~nm}$ and 520 $\mathrm{nm}$, respectively. All experiments were performed at $25^{\circ} \mathrm{C}$ with an initial starting solution of $2.0 \mathrm{ml}$ of PBS containing the desired concentration of FITC-Dextran (typically $20 \mu \mathrm{g} / \mathrm{ml}$ ) in a standard quartz spectrofluorometer cuvette. The fluorescein fluorescence intensity was first recorded at $520 \mathrm{~nm}$, then an aliquot of a solution containing the ConA conjugates was titrated into the cuvette with mixing. The fluorescein signal was recorded and this procedure was repeated for each addi- tional aliquot of ConA conjugate. Additions were made without removing the cuvette from the holder to prevent changing the cuvette alignment, and corrections were made for sample dilution. The quenching experiment was repeated a second time using the same procedure except that the $2.0 \mathrm{ml}$ starting solution of FITC-Dextran also contained $1500 \mathrm{mg} / \mathrm{dl}$ glucose. These two experiments, one with glucose and one without glucose, provided what will be called the "dynamic range envelope" for the homogeneous assay between glucose concentrations of 0 and $1500 \mathrm{mg} / \mathrm{dl}$.

\section{RESULTS}

\section{Quenching with TRITC-ConA}

Figure 3 presents a series of experiments performed to determine the extent of FITC-Dextran fluorescence quenching caused by solutions containing commercially prepared ConA conjugates, see top four curves. Results for solutions containing ConA conjugates we prepared are shown in the lower two curves. The middle two curves represent the dynamic range envelope for commercially prepared TRITC-ConA, where the lower curve of the two represents data obtained

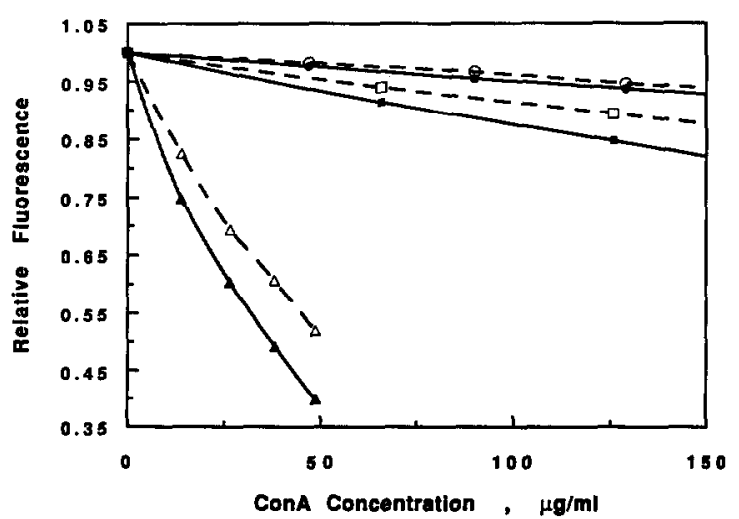

Fig. 3. Quenching of FITC-Dextran fluorescence by conjugates of TRITC-ConA and TRITC-Succ-ConA in the absence of glucose (solid lines) and in the presence (dashed lines) of $1500 \mathrm{mg} / \mathrm{dl}$ glucose. $\square$ and $\square$, commercially prepared TRITC-ConA. - and $O$, commercially prepared TRITCSucc-ConA. and $\Delta$, synthesized TRITC-Succ-ConA having 6.2 and 2.8 molecules of TRITC and succinate, respectively, per pConA. 
in the absence of glucose, and the upper curve of the two represents data obtained in the presence of $1500 \mathrm{mg} / \mathrm{dl}$ glucose. Comparing these two curves indicates that the fluorescence of a solution initially containing $125 \mu \mathrm{g} / \mathrm{ml}$ TRITC-ConA, $2.5 \mu \mathrm{g} / \mathrm{ml}$ FITC-Dextran, and no glucose, would increase by approximately $6 \%$ when $1500 \mathrm{mg} / \mathrm{dl}$ glucose was added to the solution. Therefore, one would expect only about a $6 \%$ increase in signal for a sensor based on the commercially prepared TRITC-ConA conjugate over a glucose concentration range of 0 to $1500 \mathrm{mg} / \mathrm{dl}$.

The upper two curves in Fig. 3 are the fluorescence quenching results in the absence and presence of glucose for commercially prepared TRITC-Succ-ConA for the same FITC-Dextran concentration, $2.5 \mu \mathrm{g} / \mathrm{ml}$. These curves indicate that there was not a significant increase in the FITC-Dextran fluorescence when glucose was added. A subsequent determination was made that the very narrow dynamic range envelope for TRITC-Succ-ConA was due to the fact that more than half of the commercially prepared material was inactive. Also, the protein molecules that still contained active glucose binding sites had very low dye/protein ratios which contributed to the low level of fluorescence quenching [16]. Because the results were so poor, we synthesized new ConA conjugates with the intention of improving the dynamic range of the homogeneous assay for use in the optical fiber sensor system.

Fluorescence quenching results for one of the TRITC-Succ-ConA conjugates we synthesized and subsequently purified are shown by the lower 2 curves of Fig. 3. This particular sample of TRITC-Succ-ConA had approximately 6.2 TRITC and 2.8 succinate molecules per ConA protomer. Other samples of TRITC-Succ-ConA were prepared with varying levels of TRITC and succinate conjugation, but the dynamic range envelopes were almost identical to the data presented in Fig. 3. This information helped to confirm that the conjugation and purification procedures were not having a significant effect on the glucose binding properties of the ConA. Comparison of the envelopes in Fig. 3 shows two important differences between the three samples. First, the higher dye/protein ratio obtained for the conju- gates we synthesized resulted in a significantly wider level of total fluorescence quenching. Secondly, the dynamic range envelope was significantly greater for the synthesized TRITC-SuccConA when compared with either of the commercially prepared materials at the same ConA concentration ( $30 \%$ versus $3 \%$ and $1 \%$, respectively at $50 \mu \mathrm{g} / \mathrm{ml}$ of ConA). Both of these improvements are indicative of greater specificity in the binding interactions and more efficient energy transfer which helped to significantly increase the sensitivity of the optical fiber sensor.

A series of fluorescence recovery experiments were performed over a glucose concentration range of 0 to $1500 \mathrm{mg} / \mathrm{dl}$ in order to demonstrate the reversibility of the Dextran/ConA binding interactions of the conjugates we prepared, Fig. 4. All samples had an initial FITC-Dextran concentration of $2.5 \mu \mathrm{g} / \mathrm{ml}$ and the degree of TRITC labeling ranged from 6.4 to 7.6 molecules per pConA and succinate ranged from 0 to 2.8 molecules per pConA. From these results, it was again clear that the Dextran/ConA binding was reversible and that there was relatively little effect of TRITC or succinate on the assay performance which in turn indicated that the conjugation procedures did not significantly affect the tertiary structure of ConA.

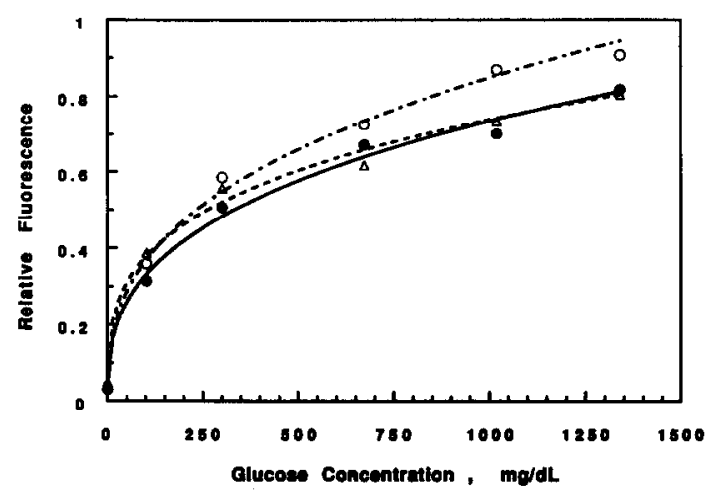

Fig. 4. Effect of conjugation on the recovery of quenched FITC-Dextran fluorescence during glucose titration. (all samples initially had $2.5 \mu \mathrm{g} / \mathrm{ml}$ FITC-Dextran). $\bullet$, ConA concentration $0.2 \mathrm{mg} / \mathrm{ml}: 6.4$ TRITC and 0 succinate molecules per pConA. $\triangle$, ConA concentration $0.24 \mathrm{mg} / \mathrm{ml}: 7.6$ TRITC and 1.2 succinate molecules per pConA. $O$, ConA concentration $0.48 \mathrm{mg} / \mathrm{ml}: 6.2$ TRITC and 2.8 succinate molecules per pConA. 


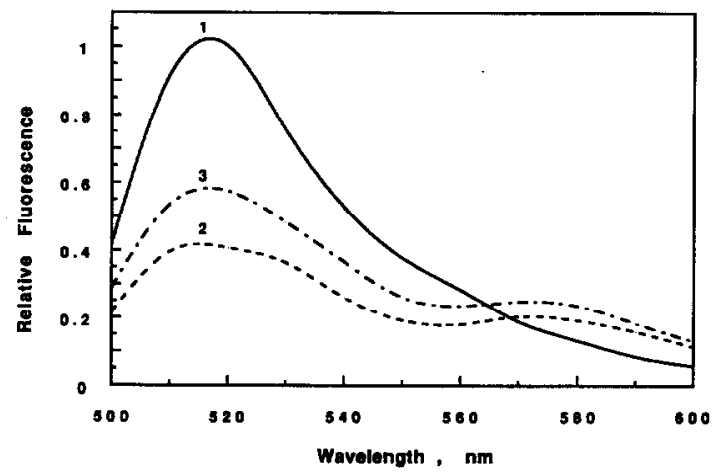

Fig. 5. The fluorescence spectra for FITC-Dextran/TRITCConA assay solutions with excitation at $485 \mathrm{~nm}$. Curve 1 is for $2.5 \mu \mathrm{g} / \mathrm{ml}$ FITC-Dextran in PBS. Curve 2 is for a solution of $2.5 \mu \mathrm{g} / \mathrm{ml}$ FITC-Dextran and $50 \mu \mathrm{g} / \mathrm{ml}$ of TRITC-SuccConA. Curve 3 is for a solution of $2.5 \mu \mathrm{g} / \mathrm{ml} \mathrm{FITC-Dextran,}$ $50 \mu \mathrm{g} / \mathrm{ml}$ of TRITC-Succ-ConA, and $1500 \mathrm{mg} / \mathrm{dl}$ of glucose.

Clearly the results in Figs. 3 and 4 indicate that the homogeneous assay system can specifically and reversibly respond to changes in glucose concentration up to $1500 \mathrm{mg} / \mathrm{dl}$. However, to fully utilize all of the advantages of the system one must be able to recalibrate the instrumentation system to account for drift without the use of external glucose calibration standards. In Fig. 5, the fluorescence spectra are plotted for three solutions when the excitation wavelength was 480 $\mathrm{nm}$. The first curve in Fig. 5 is the fluorescence emission spectra centered at $520 \mathrm{~nm}$ of a solution containing $2.5 \mu \mathrm{g} / \mathrm{ml}$ of FITC-Dextran. Curve 2 is the same solution after $50 \mu \mathrm{g} / \mathrm{ml}$ of the synthesized TRITC-Succ-ConA is added. Due to the energy transfer between FITC and TRITC when the Dextran/ConA bound complex is formed, the fluorescence signal is quenched by approximately $60 \%$. A significant portion of this signal is recovered upon addition of $1500 \mathrm{mg} / \mathrm{dl}$ glucose, curve 3. From these results, one should expect the sensor system described in Fig. 2 to be able to monitor changes in the glucose concentration by measuring changes in the FITC signal when using $480 \mathrm{~nm}$ excitation light. To obtain an internal calibration signal, the fluorescence signal from TRITC can be selectively monitored using an excitation source of $540 \mathrm{~nm}$.

\section{Response of optical sensor system}

Before the homogeneous assay system was tested in the optical fiber sensor configuration, the sensitivity of the dual wavelength instrumentation system shown of Fig. 2 was evaluated using FITC-Dextran standard solutions for a period of two seconds with a duty cycle of 0.4 , Fig. 6 . By optimizing the duty cycle and period, fluorescence bleaching was prevented. Data collection continued for at least one minute, then the average was determined. The enlarged section in Fig. 6 shows that the response was quite linear up to $10 \mu \mathrm{g} / \mathrm{ml}\left(r^{2}=0.99995\right)$, then there was a slight curvature at the higher concentration ranges probably due to the so called inner filter effect. If the fluorescein detection limit of the sensor were defined as the concentration at which the signal is twice the background noise level, then the detection limit for this dual fiber system was approximately $0.05 \mu \mathrm{g} / \mathrm{ml}$. For comparison, the sensitivity of the single fiber system developed by Schultz et al. [12] was tested in solutions of 70000 MW FITC-Dextrans sourced from four different suppliers. For all four suppliers, the detection limit was approximately $4 \mu \mathrm{g} / \mathrm{ml}$ which is almost 100 times higher than would be obtained using the dual fiber system. An explanation for the drastically improved sensitivity of the dual fiber system was that the fluorescence signals did not return through the same fiber/connector pathway as the excitation light. By isolating the excitation and emission light signals from each other,

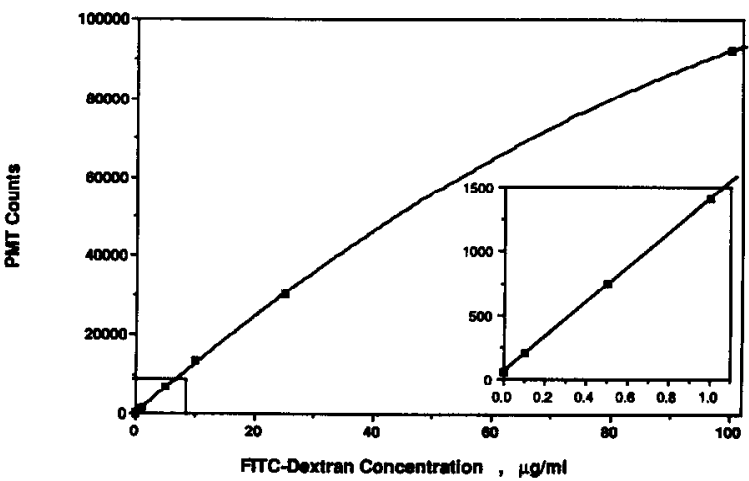

Fig. 6. Sensitivity of the dual optical fiber sensor system for 70000 MW FITC-Dextran. 


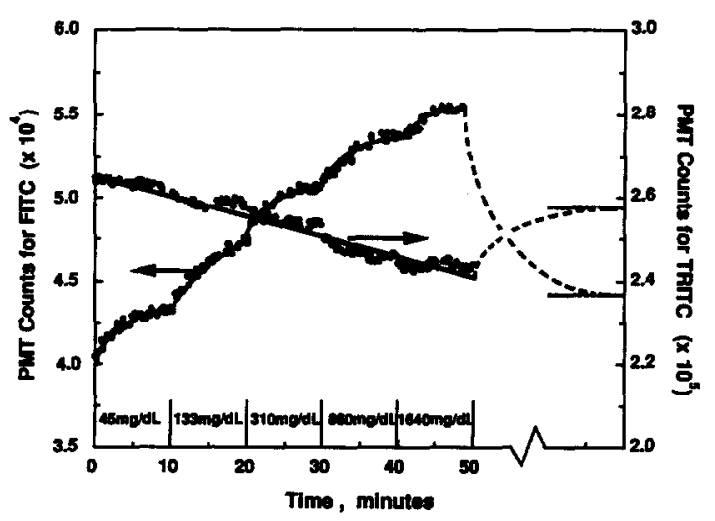

Fig. 7. Response of the dual optical fiber sensor to step changes in the glucose concentration. Glucose concentrations were monitored by changes in the FITC signal shown along the left $y$-axis while the internal calibration signal from TRITC is shown along the right $y$-axis. Glucose concentrations have been identified along the $x$-axis. The dashed horizontal lines indicate the new baseline signals after the sensor was inserted into PBS.

the background noise level of the dual fiber system was significantly reduced.

A sensor was then constructed with the dual fiber system using a solution containing $50 \mu \mathrm{g} / \mathrm{ml}$ of the synthesized TRITC-Succ-ConA conjugate and $2.5 \mu \mathrm{g} / \mathrm{ml}$ of FITC-Dextran. The sensitivity and signal response of the sensor was examined using a series of glucose standards, Fig. 7. Every $10 \mathrm{~min}$ the sensor was placed into new calibration solutions containing increasing amounts of glucose up to $1640 \mathrm{mg} / \mathrm{dl}$. After the $50 \mathrm{~min}$ time point, the sensor was returned to a PBS solution and the sensor was allowed to equilibrate for approximately $60 \mathrm{~min}$. The new baseline signal was measured as indicated by the dashed horizontal line near the right side axis in Fig. 7. Even after these data were corrected for slight drifts in the electronic components using the TRITC reference signal, the baseline had drifted up almost $30 \%$ in the two hours since the expcrimcnt began. The same sensor was again placed into a solution of $1640 \mathrm{mg} / \mathrm{dl}$ glucose, and the PMT counts increased only to about 55000 indicating significant loss of dynamic range. The dynamic range continued to decrease with time until there was virtually no glucose response after twelve hours. When the sensor was disassembled, micro- scopic examination showed that the protein appeared to have adsorbed to the interior wall of the polysulfone membrane. It was concluded that the hydrophobic polysulfone membrane had a strong tendency to adsorb proteins, and it should be replaced with a more hydrophilic membrane, e.g., cellulose acetate. This work also indicated that the response time of a sensor based on the $0.5 \mathrm{~mm}$ i.d. polysulfone fiber was approximately ten minutes and the relative dynamic range was virtually the same as obtained for the cuvette experiments presented in Fig. 4. An important observation from these results is that the response of the homogeneous assay scales directly from the cuvette experiments to the sensor experiments. This should significantly shorten the time required to evaluate an assay system for use in the optical fiber sensor.

\section{DISCUSSION}

Biosensors capable of making very reliable routine measurements for a broad range of analytes, e.g., drugs, metabolites, and hormones would be a tremendous benefit to the areas of clinical medicine and biochemical process engineering. To this end, we have developed a homogeneous assay system based on fluorescence energy transfer for use in an optical fiber sensor that can detect glucose over a broad concentration range. The sensitivity of the assay using commercially prepared ConA conjugates was quite poor, but when a new succinylation procedure was used to prepare TRITC-ConA conjugates, the sensitivity and dynamic range of the assay were both significantly improved. These improvements were attributed to two factors: more than half of the commercial material was inactive, and the dye/protein ratio was too low to achieve high energy transfer efficiencies.

The chemical modification technique for stabilizing ConA using succinic anhydride proved to be successful in preventing ConA aggregation. This was essential for the homogeneous assay to perform properly. However, it was subsequently shown that there is a sub-population of SuccConA molecules (approximately $60 \%$ of the total) 
that slowly lose their glucose binding activity over a period of several days when stored at $37^{\circ} \mathrm{C}$. Even though the majority of Succ-ConA molecules loose their ability to bind glucose, protein aggregates are not formed by the deactivated material [16]. The remaining $40 \%$ of the population resists binding site deactivation and aggregation even when stored at $37^{\circ} \mathrm{C}$ for several weeks. Future work on the assay system should focus on isolating the more stable sub-population for use in sensors to help ensure good long-term stability.

When the homogeneous assay was used in the optical fiber sensor, another stability problem arose. Adsorption of the ConA conjugates to the inner wall of the hollow fiber made the baseline and dynamic range drift with time. It is possible that membranes made of more hydrophilic polymers such as regenerated cellulose or cellulose acetate would help to reduce the amount of adsorption. Obviously, until stability of the ConA can be ensured during the life of the sensor, the inherent advantages of the internal calibration system can not be fully realized.

In spite of the ConA adsorption problem, preliminary testing was completed to evaluate the performance of the homogeneous assay in the sensor system. The time response was found to be significantly faster for a homogeneous assay sensor than for the heterogeneous sensor developed by Schultz et al. [12]. If the diffusion constant for glucose through polysulfone was assumed to be the same as the diffusion constant through the hollow fibers used by Schultz et al. $\left(3 \times 10^{-6}\right.$ $\left.\mathrm{cm}^{2} / \mathrm{s}\right)$, then a homogeneous sensor with the same diameter as the Schultz heterogeneous sensor would have a time response of approximately $1.5 \mathrm{~min}$ as compared to their reported value of $\mathbf{1 0}$ min. The significant improvement in the time response is probably attributable to the fact that the diffusion barrier from the immobilized ConA was eliminated. An added feature of the homogeneous assay sensor was that physical damage to the ConA from the immobilization procedure did not occur as was the case with the heterogeneous sensor.

Using a homogeneous energy transfer assay in an optical fiber biosensor had other advantages. Multiplexing two excitation sources made it possi- ble to measure analyte concentrations while at the same time the sensor could be internally recalibrated to correct for instrumentation drifts. Also, the optimum design criteria for a specific analyte concentration range were more easily determined for the sensor because the sensitivity and dynamic range for the fluorescence quenching assay scaled down directly from the cuvette scale to the optical fiber sensor scale.

There are several design variables one can adjust to optimize the assay for a particular concentration range [23]. The bioreceptor can be changed to alter the binding constant. Also, the concentration of the bioreceptor and competing ligand can be adjusted. Finally, the efficiency of energy transfer can be changed by using donor /acceptor pairs that have different degrees of spectral overlap. Earlier work clearly showed that the donor/acceptor pair should be carefully selected in order to achieve a high energy transfer efficiency, and the donor and acceptor should have good separation between the excitation and emission spectra (Stokes shift). The large Stokes shift makes it easier to isolate the donor and acceptor fluorescence signal for sensor measurements. All of these design parameters help to make the homogeneous assay system quite versatile for other analytes.

The optical fiber sensor does have a practical lower limit of detection. Using the monovalent ligand/receptor molecular model [23], we estimated the lower detection limit for a given bioreceptor/competing ligand pair over a specific analyte concentration range. If one makes the following assumptions: (1) there is an increase in fluorescence of $35 \%$ over the analyte concentration range; (2) the mole ratio of competing ligand to bioreceptor is 1 to 10 ; (3) the competing ligand concentration is $10 \mathrm{nM}$; (4) the equilibrium constants of analyte and competing ligand for the bioreceptor are the same and equal to $10^{8} \mathrm{M}^{-1}$, then it should be possible to detect analyte concentrations between $10^{-8}$ and $10^{-7} \mathrm{M}$ with an adequate signal response using the dual fiber system. Because these design variables can have a wide range of values, the detection range can be quite easily shifted to the desired analyte concentration range by adjusting the design parameters. 
In summary, we believe the optical fiber sensor system described here has several inherent advantages that can be capitalized on by carefully designing the homogeneous assay conditions to match the required assay conditions.

The authors wish to thank Dr. Thomas Stevenson, Evangeline Caesar, Maher Siraj, and Ed Ullman for their significant contributions to the completion of this work.

\section{REFERENCES}

1 J. Janata, Anal. Chem., 62 (1990) 33R.

2 J.N. Roe, Pharm. Res., 9 (1992) 835.

3 G.A. Rechnitz, J. Clin. Lab Anal., 1 (1987) 308.

4 R.K. Kobos, Trends Anal. Chem., 6 (1987) 6.

5 T.E. Edmonds (Ed.), Chemical Sensors, Chapman and Hall, New York, 1988.

6 R.C. Hughes, A.J. Ricco, M.A. Butler and S.J. Martin, Science, 254 (1991) 74.

7 L.A. Saari, Trends Anal. Chem., 6 (1987) 85.

8 O.S. Wolfbeis (Ed.), Fiber Optic Chemical Sensors and Biosensors, CRC Press, Boca Raton, FL, 1991.

9 W.R. Seitz, in A.P.F. Turner, I. Karube, G.S. Wilson
(Eds.), Biosensors: Fundamentals and Applications, Oxford University Press, New York, 1987, Chap. 30.

10 D.L. Meadows and J.S. Schultz, Talanta, 35 (1988) 145.

11 S. Mansouri and J.S. Schultz, Bio/Technology, October (1984) 885.

12 J.S. Schultz, S. Mansouri and I.J. Goldstein, Diabetes Care, 5 (1982) 245.

13 E.F. Ullman, M. Schwarzberg and K.E. Rubenstein, J. Biol. Chem., 251 (1975) 4172.

14 L. Stryer, Ann. Rev. Biochem., 47 (1978) 819.

15 P.L. Khanna and E.F. Ullman, Anal. Biochem., 108 (1980) 156.

16 D.L. Meadows, PhD Dissertation, A Fiber Optic Biosensor for Glucose Monitoring Based on Fluorescence Energy Transfer, University of Michigan, Ann Arbor, MI, 1988.

17 D.L. Meadows and J.S. Schultz, Proc. SPIE (Fiber Optic Medical and Fluorescent Sensors and Applications), 1648 (1992) 202.

18 J.L. Wang, G.R. Gunther, and G.M. Edelman, in H. Bittinger and H.P. Schnebli (Eds.), Concanavalin $A$ as a Tool, Wiley, New York, 1976, Chap. 57.

19 B.B.L. Agrawal, I.J. Goldstein, G.S. Hassing and L.L. So, Biochemistry, 7 (1968) 4211.

20 N.M. Young, Biochim. Biophys. Acta., 336 (1974) 46.

21 G.R. Gunther, J.L. Wang, I. Yahara, B.A. Cunningham and G.M. Edelman, Proc. Nat. Acad. Sci., 70 (1973) 1012.

22 D.L. Meadows, J.S. Shafer and J.S. Schultz, J. Immunol. Methods, 143 (1991) 263.

23 D.L. Meadows and J.S. Schultz, Biotechnol Bioeng., 37 (1991) 1066. 